\title{
ETS family-associated gene fusions in Japanese prostate cancer: analysis of 194 radical prostatectomy samples
}

\author{
Yohei Miyagi ${ }^{1}$, Takeshi Sasaki ${ }^{2}$, Kiyoshi Fujinami ${ }^{3}$, Jinyu Sano ${ }^{4}$, Yutaka Senga ${ }^{3}$, \\ Takeshi Miura $^{5}$, Yoichi Kameda ${ }^{6}$, Yuji Sakuma ${ }^{1}$, Yoshiyasu Nakamura ${ }^{1}$, Masaoki Harada ${ }^{1}$ \\ and Eiju Tsuchiya ${ }^{1}$ \\ ${ }^{1}$ Molecular Pathology and Genetics Division, Kanagawa Cancer Center Research Institute, Yokohama, Japan; \\ ${ }^{2}$ Department of Pathology, Yokohama City University Medical Center, Yokohama, Japan; ${ }^{3}$ Department of \\ Urology, Chigasaki Municipal Hospital, Chigasaki, Japan; ${ }^{4}$ Department of Pathology, Chigasaki Municipal \\ Hospital, Chigasaki, Japan; ${ }^{5}$ Department of Urology, Kanagawa Cancer Center Hospital, Yokohama, Japan \\ and ${ }^{6}$ Department of Pathology, Kanagawa Cancer Center Hospital, Yokohama, Japan
}

\begin{abstract}
The incidence and clinical significance of the TMPRSS2:ERG gene fusion in prostate cancer has been investigated with contradictory results. It is now common knowledge that significant variability in gene alterations exists according to ethnic background in various kinds of cancer. In this study, we evaluated gene fusions involving the ETS gene family in Japanese prostate cancer. Total RNA from 194 formalin-fixed and paraffin-embedded prostate cancer samples obtained by radical prostatectomy was subjected to reversetranscriptase polymerase chain reaction to detect the common TMPRSS2:ERG T1-E4 and T1-E5 fusion transcripts and five other non-TMPRSS2:ERG fusion transcripts. We identified 54 TMPRSS2:ERG-positive cases $(54 / 194,28 \%)$ and two HNRPA2B1:ETV1-positive cases $(2 / 194,1 \%)$. The SLC45A3-ELK4 transcript, a fusion transcript without structural gene rearrangement, was detectable in five cases $(5 / 194,3 \%)$. The frequencies of both TMPRSS2:ERG- and non-TMPRSS2:ERG-positive cases were lower than those reported for European, North American or Brazilian patients. Internodular heterogeneity of TMPRSS2:ERG was observed in 5 out of 11 multifocal cases (45\%); a frequency similar to that found in European and North American cases. We found a positive correlation between the TMPRSS2:ERG fusion and a Gleason score of $\leq 7$ and patient age, but found no relationship with pT stage or plasma prostate-specific antigen concentration. To exclude the possibility that Japanese prostate cancer displays novel TMPRSS2:ERG transcript variants or has unique $5^{\prime}$ fusion partners for the ETS genes, we performed $5^{\prime}$ RACE using fresh-frozen prostate cancer samples. We identified only the normal $5^{\prime}$ cDNA ends for ERG, ETV1 and ETV5 in fusion-negative cases. Because we identified a relatively low frequency of TMPRSS2:ERG and other fusions, further evaluation is required before this promising molecular marker should be introduced into the management of Japanese prostate cancer patients.
\end{abstract}

Modern Pathology (2010) 23, 1492-1498; doi:10.1038/modpathol.2010.149; published online 6 August 2010

Keywords: ERG; ETS; Japanese; prostate cancer; RT-PCR; TMPRSS2

Tomlins et $a l^{1}$ first reported in 2005 that recurrent genomic rearrangements are observed in prostatic

Correspondence: Dr Y Miyagi, MD, PhD, Molecular Pathology and Genetics Division, Kanagawa Cancer Center Research Institute, 1-1-2 Nakao, Asahi-ku, Yokohama 241-0815, Japan.

E-mail: miyagi@gancen.asahi.yokohama.jp

Received 5 March 2010; revised 18 May 2010; accepted 27 May 2010; published online 6 August 2010 adenocarcinomas, resulting in gene fusion between the untranslated region of the androgen-driven gene TMPRSS2 and the oncogenic transcription factor genes of the ETS family. This gene fusion is now recognized as a frequent event, occurring in almost half of prostate cancer cases. ${ }^{2}$ To date, 14 genes (TMPRSS2, SLC45A3, HERV-K, KLK2, CANT1, FOXP1, HERVK17, EST14, FLJ35294, C15orf21, HNRPA2B1, DDX5, ACSL3 and NDRG1) have been 
identified as $5^{\prime}$ fusion partners for the four ETS family transcription factor genes (ERG, ETV1, ETV4 and ETV5), resulting in $>20$ different fusion genes in prostate cancer. ${ }^{2,3,4,5}$ Among these, approximately 90\% occur between TMPRSS2 and ERG. ${ }^{2}$

The association of the TMPRSS2:ERG gene fusion with prostate cancer prognosis has been evaluated in multiple studies but with contradictory results. ${ }^{2}$ Although its clinical significance remains to be clarified, because of its high incidence, TMPRSS2:ERG is expected to be a useful marker for the early detection of prostate cancer. Evaluation of TMPRSS2:ERG transcripts in urine samples after prostatic massage in combination with other prostate cancer marker RNAs such as PAC3 are candidates for prostate cancer screening. ${ }^{6}$

It is a well-known paradigm showing importance of ethnicity that the frequency of EGFR mutation in lung adenocarcinomas significantly differs between Western and Asian countries. ${ }^{7}$ In this study, we evaluated gene fusions involving the ETS family genes in prostate cancer among the Japanese.

\section{Materials and methods}

\section{Study Population and Preparation of Prostate Cancer} Specimens

The analysis involved 194 Japanese cases of prostate cancer who had radical prostatectomy at the Chigasaki Municipal Hospital (CMH), Chigasaki, Japan (88 cases) and the Kanagawa Cancer Center Hospital (KCCH), Yokohama, Japan (106 cases). None of the patients received preoperative radiation or androgen deprivation therapy. All patients from $\mathrm{KCCH}$ and 51 patients from CMH, whose prostatectomy specimens were subjected to whole-mount pathological examination, were consecutive cases from 2003 to 2008 (KCCH) and from 2003 to 2006 $(\mathrm{CMH})$. Patients with small tumors of $<5 \mathrm{~mm}$ in the maximum diameter were excluded from the study. A total of 37 patients from $\mathrm{CMH}$, whose specimens were not subjected to whole-mount examination, were randomly selected from those operated from 1998 to 2002. The institutional review boards of both hospitals approved this study. Generally, macroscopic tumor location was mapped and, for the index tumor of each case, three serial slices of $10-\mu \mathrm{m}$ thickness were prepared from paraffinembedded specimens, from which total RNA was extracted. For the cases consisting of multiple tumor nodules, which were separated from each other by $>5 \mathrm{~mm}$ and where tumor size was $>5 \mathrm{~mm}$ maximum diameter, RNA was extracted from each nodule separately and nodules were each analyzed subsequently as index tumors.

RNA extraction was performed using Recover All total nucleic acid isolation kit (Ambion, Austin, TX, USA) according to the manufacturer's instructions.

\section{Reverse Transcriptase-Polymerase Chain Reaction (RT-PCR)}

RNA samples were first subjected to primary RTPCR using $200 \mathrm{ng}$ of sample RNA with the SuperScript III One-Step RT-PCR system (Invitrogen, Carlsbad, CA, USA) in $25 \mu$ l of reaction mixture according to the manufacturer's instructions. Then, $1.5 \mu \mathrm{l}$ of the primary reaction was subjected to secondary semi-nested PCR in $25 \mu \mathrm{l}$ of the recommended buffer. Next, $5 \mu \mathrm{l}$ of the amplified reaction was electrophoresed on a $2.5 \%$ agarose gel, followed by staining with ethidium bromide and imaging. The primer sequences are provided in Supplementary Table 1. Representative PCR product bands were recovered and subcloned into the pGem-T-easy plasmid vector (Promega, Madison, WI, USA). Nucleotide sequences were determined to confirm that the bands corresponded to the predicted fusion transcripts. The detailed experimental conditions are provided in the Supplementary Information.

\section{5'-Rapid Amplification of cDNA Ends (RACE)}

Each frozen sample was confirmed histologically to contain cancer tissue. Total RNA was extracted using TRIzol reagent (Invitrogen). First, expression of the ERG, ETV1, ETV4 and ETV5 transcripts and the control porphobilinogen deaminase $(P B G D)$ was evaluated by conventional RT-PCR, with primers located in exons not involved in reported prostate cancer gene fusions (listed in Supplementary Table 2). Then, $3 \mu \mathrm{g}$ of each total RNA was subjected to 5'-RACE with a GeneRacer kit (Invitrogen), according to the manufacturer's protocol. Amplified products were subcloned into pGem-T-easy plasmid vector (Promega) and their nucleotide sequences were determined. For RACE of ETV5, the primary RACE PCR gave no products and the nested PCR was performed with the primers given in Supplementary Table 2 . The precise experimental conditions are provided in the Supplementary Information.

\section{Statistical Analysis}

Statistical difference was evaluated using Yates' $\chi^{2}$ test or Fisher's test, and $P$-values of $<0.05$ were considered significant.

\section{Results}

Incidence of Fusion Gene Transcript-Positive Prostate Cancer Cases and Clinicopathological Characteristics

To verify the quality and integrity of the RNA samples, we first performed RT-PCR for $P B G D$, a ubiquitously expressed housekeeping gene. All RNAs used in this study gave strong and clear PCR products for $P B G D$. Then, we detected fusion gene 


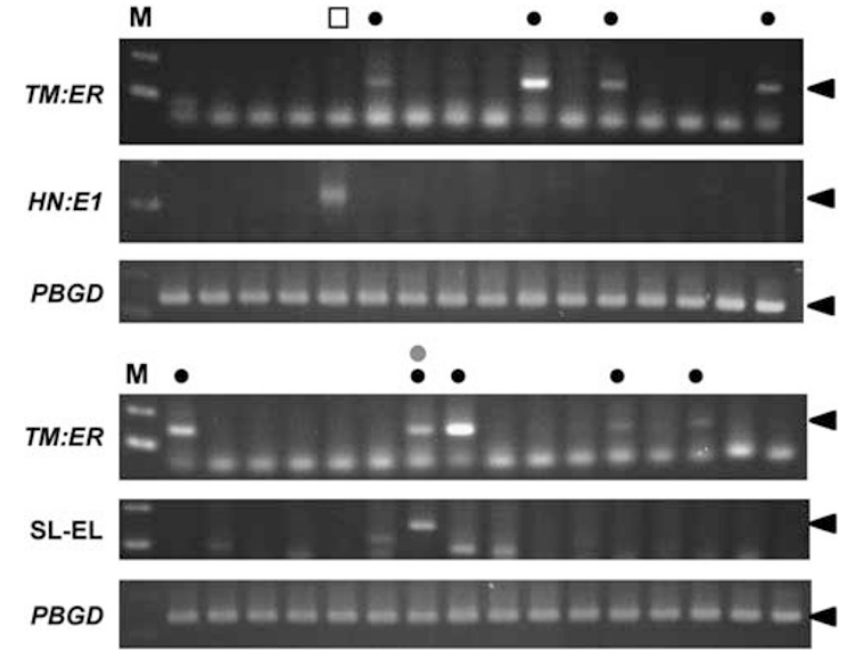

Figure 1 Representative results of RT-PCR to detect fusion transcripts. The upper panel shows a set of 16 cases demonstrating four cases positive for the TMPRSS2:ERG transcript (black circles) and one case positive for the HNRPA2B1:ETV1 transcript (open square). The lower panel shows another set of 16 cases demonstrating five cases positive for the TMPRSS2:ERG transcript and one case positive for the SLC45A3-ELK4 transcript (gray circle). $P B G D$ RT-PCR is shown at the bottom of each panel as a positive control for RNA sample integrity. M, molecular size marker; TM:ER, TMPRSS2:ERG; HN:E1, HNRPA2B1:ETV1; SL-EL, SLC45A3-ELK4.

transcripts for two TMPRSS2:ERG fusions (the T1-E4 and T1-E5 variants), TMPRSS2:ETV1, SLC45A3:ETV1, HERV-K:ETV1, Cl5ORF21:ETV1 and HNRPA2B1:ETV1. Out of 194 radical prostatectomy samples, $54(28 \%)$ were positive for the TMPRSS2:ERG T1-E4 transcript. The T1-E5 transcript was present in two cases $(1 \%)$, and both of these also contained the T1-E4 transcript. Two cases $(1 \%)$ were positive for the HNRPA2B1:ETV1 transcript. There was no overlap between the TMPRSS2:ERG-positive and HNRPA2B1:ETV-positive cases. No other fusion gene transcripts were identified. For the SLC45A3-ELK4 fusion transcript, five cases demonstrated identifiable bands after semi-nested PCR. Two of them were also positive for TMPRSS2:ERG. Representative gel photographs showing fusion transcripts are shown in Figure 1.

We next evaluated the relationship between the incidence of the TMPRSS2:ERG fusion and the Gleason score in all 194 cases. The incidence of gene fusion was significantly lower (8 out of 54 cases, $15 \%$ ) in those with a high risk Gleason score $(\geq 8)$ than in cases with low or intermediate risk Gleason score ( $\leq 7$; Table 1). For the 157 samples on which whole-mount examination was performed, the incidence of the TMPRSS2:ERG fusion was evaluated in relation to patient age, plasma prostate-specific antigen (PSA) concentration and pathological tumor (pT) stage. The incidence was significantly lower in patients aged $\leq 59$ years, or in those aged $\geq 71$ compared with those aged between 60 and 69 years. There was no significant
Table 1 Clinicopathological characteristics of TMPRSS2:ERGpositive cases

\begin{tabular}{|c|c|c|c|c|}
\hline Characteristics & $\begin{array}{c}\text { TMPRSS2: } \\
\text { ERG (-), } \\
\text { no. of cases } \\
(\%)\end{array}$ & $\begin{array}{c}\text { TMPRSS2: } \\
\text { ERG }(+), \\
\text { no. of cases } \\
(\%)\end{array}$ & Total & $\mathrm{P}$-value \\
\hline \multicolumn{5}{|l|}{ Gleason score } \\
\hline$\leq 7$ & $95(67)$ & $46(33)$ & 141 & $0.02^{\mathrm{b}}$ \\
\hline$\geq 8$ & $45(85)$ & $8(15)$ & 53 & \\
\hline Total & $140(72)$ & $54(28)$ & 194 & \\
\hline \multicolumn{5}{|l|}{ Age } \\
\hline$\leq 59$ & $17(94)$ & $1(6)$ & 18 & $0.004^{\mathrm{b}}$ \\
\hline $60-69$ & $72(64)$ & $41(36)$ & 113 & \\
\hline$\geq 70$ & $24(92)$ & $2(8)$ & 26 & \\
\hline Total & $113(72)$ & $44(28)$ & 157 & \\
\hline \multicolumn{5}{|l|}{$P T$} \\
\hline pT2 (organ confined) & $55(71)$ & $22(29)$ & 77 & 0.99 \\
\hline pТ3a (EPE) & $43(72)$ & $17(28)$ & 60 & \\
\hline pT3b (s.v. invasion) & $15(75)$ & $5(25)$ & 20 & \\
\hline Total & $113(72)$ & $44(28)$ & 157 & \\
\hline \multicolumn{5}{|l|}{$P S A$} \\
\hline 4 to $<10 \mathrm{ng} / \mathrm{ml}$ & $58(69)$ & $26(31)$ & 84 & 0.42 \\
\hline 10 to $20 \mathrm{ng} / \mathrm{ml}$ & $40(71)$ & $16(29)$ & 56 & \\
\hline $20 \mathrm{ng} / \mathrm{ml}<$ & $15(88)$ & $2(12)$ & 17 & \\
\hline Total & $113(72)$ & $44(28)$ & 157 & \\
\hline
\end{tabular}

${ }^{\mathrm{a}}$ Gleason score was analyzed against all 194 cases examined. Age, pT and PSA were analyzed against 157 cases for whom the whole-mount examination was performed for the total prostatectomy specimen and the pathological stage was exactly determined.

$\mathrm{b}$ The statistical difference was evaluated using Yates' $\chi^{2}$ test and $P<0.05$ was considered as significant.

correlation with either pT stage or PSA concentration (Table 1). The two HNRPA2B1:ETV1-positive cases were high-grade adenocarcinoma with a Gleason score of 8 and 9, respectively.

For the 157 whole-mount cases, the incidence of the TMPRSS2:ERG fusion was also separately evaluated for transition zone and non-transition zone cancers. In all, $26(17 \%)$ were recognized as transition zone cancers. The TMPRSS2:ERG-positive case was $27 \%$ (35 out of 131) in non-transition zone and $23 \%$ (6 out of 26 ) in transition zone cancers, and the incidence was not significantly different between them.

\section{Internodular Differences in the Incidence of TMPRSS2:ERG}

In all, 11 cases matched our criteria for multiple nodules. Of these, seven cases consisted of two nodules and four cases had three nodules (Table 2). Out of 11 cases, $6(55 \%)$ were positive for TMPRSS2:ERG transcripts in at least one nodule. When we evaluated the incidence per nodule, rather than per case, 8 out of 26 nodules (31\%) were positive for the fusion transcripts. There was no significant difference between multiple cases of nodules and non-multiple cases in the incidence 
Table 2 TMPRSS2:ERG fusion transcripts in cases with multiple tumor nodules

\begin{tabular}{|c|c|c|c|c|}
\hline $\begin{array}{l}\text { Nodules } \\
\text { pattern }\end{array}$ & $\begin{array}{c}\text { No. of } \\
\text { case }\end{array}$ & $\begin{array}{c}\text { Nodule } \\
\text { no. }\end{array}$ & & $\begin{array}{c}\text { Fusion } \\
\text { positive (\%) }\end{array}$ \\
\hline$\bigcirc \bigcirc$ & 2 & 4 & Per case & $6 / 11(55)$ \\
\hline$\bigcirc$ & 4 & 8 & Per nodule & $8 / 26(31)$ \\
\hline & 1 & 2 & & \\
\hline$\bigcirc \bigcirc \bigcirc$ & 3 & 9 & & \\
\hline$\bigcirc \bigcirc 0$ & - & - & & \\
\hline & 1 & 3 & & \\
\hline & - & - & & \\
\hline Total & 11 & 26 & & \\
\hline
\end{tabular}

Fusion-negative nodule.

- Fusion-positive nodule.

of TMPRSS2:ERG. Internodular heterogeneity was observed in $45 \%(5 / 11)$ of cases.

\section{Detection of ERG, ETV1 and ETV5 5-Fusion Partners}

We performed 5'-RACE on eight frozen prostate cancer tissues that did not contain any of the fusion gene transcripts examined in this study, together with two positive controls: one positive for TMPRSS23:ERG and one positive for HNRPA2B1:ETV1. First, we examined ERG, ETV1, ETV4 and ETV5 expression by conventional RT-PCR with primers located in separate exons that have not been reported to be involved in prostate cancer gene fusions. Considerable amounts of ERG, ETV1 and ETV5 transcripts were detected in all samples, but the levels of ETV4 were low in all cases (Figure 2a). Therefore, 5'-RACE was performed for ERG, ETV1 and ETV5 only. The quality of each $5^{\prime}$-RACE template was verified by amplifying approximately 1 -kbp fragments of the $5^{\prime}$-end of the $\beta$-actin cDNA. For RACE of ETV5, the primary RACE PCR gave no products and hence nested PCR was performed with the primers given in Supplementary Table 2. The RACE products were sequenced to verify their identity. The expected 5'-RACE bands derived from rearranged TMPRSS2:ERG and HNRPA2B1:ETV1 fusion transcripts were demonstrated in the positive control samples (Figure 2b, panels for ERG and ETV1). Major 5'-RACE bands observed in the eight experimental samples were derived from normal non-fusion transcriptions of ERG, ETV1 and ETV5 (Figure 2b). Although extra bands derived from normal but alternatively initiated transcripts were identified with some nonspecific bands of PCR artifacts (Figure 2b, sequencing data not shown), no $5^{\prime}$ novel or unexamined fusion partners were identified.

\section{Fusion Transcripts between $S L C 45 A 3$ and ELK4 in Frozen Samples}

Expression of fusion transcripts between SLC45A3 and ELK4 was evaluated in the frozen prostate
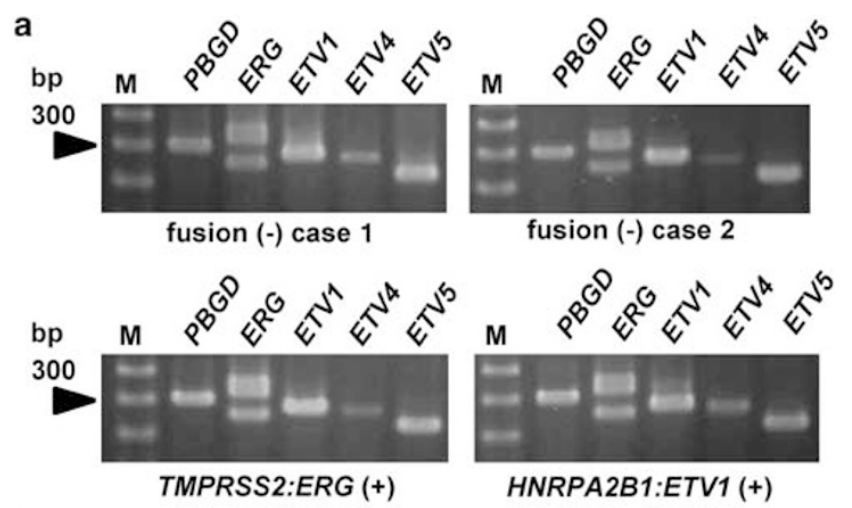

b

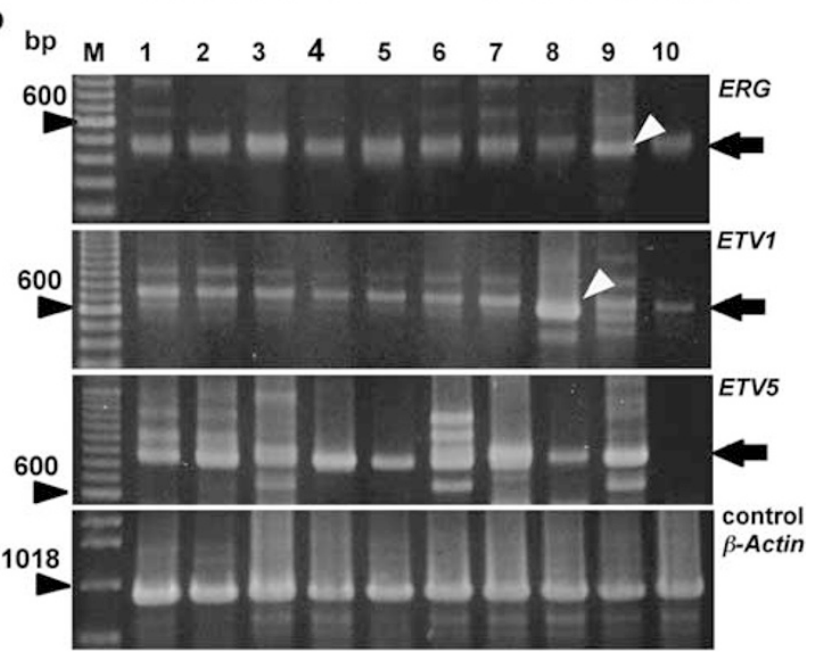

Figure 2 The expression of and 5'-RACE for ERG, ETV 1 and ETV5 transcripts. (a) RT-PCR showing the presence of ERG, ETV1, ETV4 and ETV5 transcripts. The upper two panels show the results for two representative fusion-negative cases, and the lower panels show those for fusion-positive cases as indicated. PBGD RT-PCR results are shown as a positive control. (b) $5^{\prime}$-RACE PCR. The upper three panels show 5'-RACE for ERG, ETV1 and ETV5, respectively. The bottom panel shows $5^{\prime}$-RACE for $\beta$-actin, as a template quality control. Bands indicated by white arrowheads correspond to the $5^{\prime}$ ends of the TMPRSS2:ERG fusion transcript (lane 9) and the HNRPA2B1:ETV1 fusion transcript (lane 8), respectively, as confirmed by DNA sequencing. Black arrows in the right side indicate the bands derived from normally initiated non-fusion transcripts of ERG, ETV1 or ETV5 in each panel. $\mathrm{bp}$, base pairs; $\mathrm{M}$, molecular size marker.

cancer samples used for RACE. After primary RTPCR, a strong band for the SLC45A3-ELK4 transcript was obtained in two cases, one of which was also positive for the HNRPA2B1:ETV1 fusion. After amplification by semi-nested PCR, all but one case showed the SLC45A3-ELK4 transcript (Figure 3).

\section{Discussion}

In this study, we reported the incidence of the TMPRSS2:ERG fusion to be $28 \%$ in Japanese prostate cancer samples obtained from radical prostatectomy specimens. Although a very high incidence $(\geq 70 \%)$ has been reported in some small-scale RT-PCR studies, in studies of $>50$ cases, 
the incidence is 40-60\% (mean 53\%) when restricted to radical prostatectomy specimens (Table 3). All the prostate cancer patients involved in these other studies were collected in Europe, North America or Brazil, without detailed ethnic information. The Japanese incidence of $28 \%$ is relatively low compared with these studies. Only one study, from Mosquera et $a l^{8}$ that examined 100 prostate biopsy specimens from patients in the United States, reported the TMPRSS2:ERG incidence separately in Caucasian and non-Caucasian patients. Although the incidence was $46 \%$ (46/100) overall, it was significantly different in Caucasians (44/85, $52 \%)$ and in non-Caucasians $(2 / 15,13 \%)$. This may

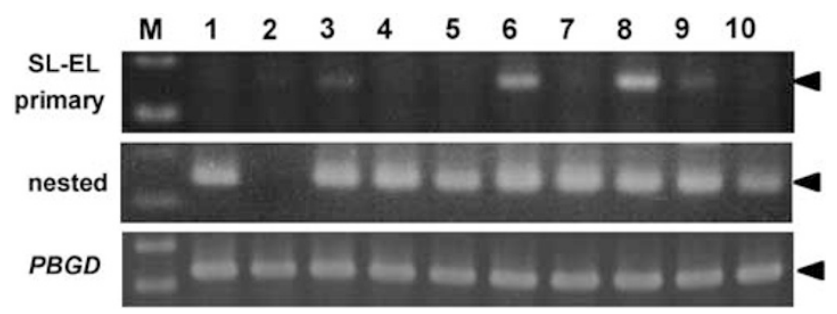

Figure 3 Expression of SLC45A3-ELK4 fusion transcripts in frozen prostate cancer samples with or without other fusion transcripts. The same sample set used for $5^{\prime}$-RACE was evaluated by RT-PCR to detect SLC45A3-ELK4 fusion transcripts. The upper panel shows the primary PCR, the middle panel shows the seminested PCR and the lower panel shows PBGD RT-PCR for template quality control. The sample numbers shown at the top correspond to those in Figure 2. M, molecular size marker. imply that the difference in incidence resulted from different genetic backgrounds rather than the effects of lifestyle or diet. Therefore, it is possible that the low incidence among the Japanese is at least partly because of the genetic background of the patients.

Low TMPRSS2:ERG incidence can be partly associated with the characteristics of the sample population. In two Swedish watchful waiting cohorts, the incidence was $15 \%(17 / 111)$ and $18 \%$ $(62 / 354)$, respectively. ${ }^{9,10}$ In these studies, the prostate cancer samples were obtained by transurethral resection of the prostate (TURP), representing mainly transition zone carcinomas. The included patients were restricted to those at early clinical stages (T1a-b, Nx, M0). From these studies, the incidence of the TMPRSS2:ERG fusion seems to be less frequent in patients at early clinical stages and in prostate cancers obtained by TURP, even among European patients. However, our Japanese prostate cancer samples were obtained by radical prostatectomy, and the low incidence may not be because of the sample characteristics.

So far, 17 types of fusion transcripts have been observed for the TMPRSS2:ERG rearrangement. ${ }^{11}$ Variation in the transcripts is generally caused by alternative splicing of the fusion transcript, and the most common transcript is a recombination between exon 1 of TMPRSS2 and exon 4 of ERG, designated T1-E4. Variants T1-E4 and T1-E5, those evaluated by RT-PCR in this study, are reported to correspond to 89-99\% of TMPRSS2:ERG fusion transcripts. ${ }^{11,12,13}$

Table 3 Prevalence of the TMPRSS2:ERG fusion in prostate cancer ${ }^{\mathrm{a}}$

\begin{tabular}{|c|c|c|c|c|c|}
\hline Sample & Incidence (\%) & Assay method & Fusion type & Patient residence & Reference \\
\hline PCa, rp & $58 / 118(49)$ & FISH & & Germany, USA & 19 \\
\hline $\mathrm{PCa}, \mathrm{rp}$ & $35 / 59(59)$ & RT-PCR & \multirow[t]{2}{*}{8 types } & USA & 12 \\
\hline PCa, rp & $120 / 253(47)$ & FISH & & USA, Canada, Germany & 21 \\
\hline PCa, rp/LN & $38 / 63(60)(70)^{\mathrm{b}}$ & RT-PCR/FISH & \multirow[t]{2}{*}{ T1-E4, iso 2} & USA, Sweden & 22 \\
\hline PCa, rp & $30 / 56(54)$ & FISH & & USA & 23 \\
\hline $\mathrm{PCa}, \mathrm{rp}$ & $81 / 165(49)$ & RT-PCR & \multirow[t]{7}{*}{ T1-E4/T2-E4 } & Canada & 26 \\
\hline PCa, TURP/en & $17 / 111(15)$ & RT-PCR/FISH & & Sweden $^{c}$ & 9 \\
\hline PCa, TURP & $134 / 445(30)$ & FISH & & UK & 27 \\
\hline PCa, TURP & $62 / 354$ (18) & DASL & & Sweden $^{\mathrm{d}}$ & 10 \\
\hline $\mathrm{PCa}, \mathrm{rp}$ & $41 / 101(41)$ & DASL & & USA & 10 \\
\hline PCa, rp & $60 / 125(48)$ & FISH & & Brazil & 28 \\
\hline PCa, rp & $217 / 521(42)$ & FISH & & USA & 29 \\
\hline
\end{tabular}

PCa, prostate cancer; rp, radical prostatectomy; FISH, fluorescence in situ hybridization; LN, lymph node; iso 2, TMPRSS2 isoform 2; TURP, transurethral resection of the prostate; en, transvesical enucleation of adenoma; DASL, DNA (cDNA)-mediated annealing, selection, ligation, and extension assay.

${ }^{\mathrm{a}}$ Studies involving $>50$ cases are listed.

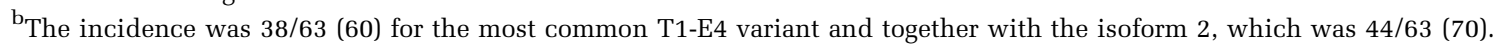

${ }^{\mathrm{c}}$ Watchful waiting cohort.

${ }^{\mathrm{d}}$ Five Swedish watchful waiting cohorts, partly overlapping with Demichelis et al. ${ }^{9}$

${ }^{\mathrm{e}}$ Familial prostate cancers. 
Therefore, the low incidence among the Japanese is not likely to be because of the assays chosen.

In addition to the most common TMPRSS2:ERG fusion, we also evaluated five other fusion transcripts: TMPRSS2:ETV1, SLC45A3:ETV1, HERVK:ETV1, Cl5ORF21:ETV1 and HNRPA2B1:ETV1. Only two cases $(1 \%, 2 / 194)$ were positive for HNRPA2B1:ETV1 transcripts, and no other fusion transcripts were identified. Non-TMPRSS2:ERG fusion is reported to be rare, and in total accounts for only $10 \%$ of all fusions. ${ }^{2}$ Although we did not evaluate all known non-TMPRSS2:ERG fusion transcripts, non-TMPRSS2:ERG fusion seems to be less frequent in Japanese prostate cancer patients than in other prostate cancer patients, similar to our findings for TMPRSS2:ERG. To exclude the possibility that Japanese prostate cancer exhibits a novel TMPRSS2:ERG transcript variant, or has unique $5^{\prime}$ fusion partners for the ETS genes, we performed 5'-RACE. We did not detect any novel gene fusions.

The combination of SLC45A3 exon 1 and the ETS family gene ELK4 exon 2 is a known fusion transcript without gene rearrangement that is observed in both normal and malignant prostatic tissues, but is overexpressed in some prostatic cancers. ${ }^{14,15}$ In light of the low incidence of the TMPRSS2:ERG fusion, it is possible that there is instead an increased incidence of SLC45A3-ELK4 overexpression among Japanese prostate cancer patients. In our study, detectable expression of the SLC45A3-ELK4 fusion transcript in formalin-fixed and paraffin-embedded specimens, which may correspond to overexpression, was rare, and therefore this is probably not a major molecular cause of prostate cancer in the Japanese.

The multifocality of prostate cancer has been recognized both morphologically and genetically. ${ }^{16,17}$ Mehra et $a l^{18}$ first reported that the TMPRSS2:ERG fusion could be heterogeneous in multifocal prostate cancer. ${ }^{28}$ There can be heterogeneity between different tumor foci, not only in the presence or absence of the fusion, but also in the mechanism of the fusion, that is, deletion or translocation. This interfocal or internodular heterogeneity has been confirmed by several follow-up studies. ${ }^{11}$ In this study, we analyzed 11 multifocal cases and found internodular heterogeneity in 5 of them (45\%), demonstrating that the TMPRSS2:ERG genetic heterogeneity is not different between the Japanese and the European or the US studies.

The prognostic utility of the TMPRSS2:ERG fusion has been evaluated in multiple studies but with contradictory results. We found a positive correlation between the TMPRSS2:ERG fusion and a Gleason score of $\leq 7$, but found no relationship with pT stage or PSA concentration. Further studies of clinical outcome, with a larger number of patients, are needed to assess the clinical significance of the fusion gene in Japanese prostate cancer.

In this study, we have raised the possibility that Japanese prostate cancer patients exhibit gene fusion events involving the ETS family genes less frequently than European or North American prostate cancer patients. Further studies with large patient cohorts, and also studies on normal and precursor lesions such as prostatic intraepithelial neoplasia, are needed before an analysis of the TMPRSS2:ERG and other fusions should be introduced into cancer screening programs and the management of prostate cancer in the Japanese.

\section{Acknowledgements}

We thank Ms Yukiko Yamazaki and Mitsuyo Yoshihara at the Molecular Pathology and Genetics Division, Kanagawa Cancer Center Research Institute, for their technical assistance.

\section{Disclosure/conflict of interest}

The authors declare no conflict of interest.

\section{References}

1 Tomlins SA, Rhodes DR, Perner S, et al. Recurrent fusion of TMPRSS2 and ETS transcription factor genes in prostate cancer. Science 2005;310:644-648.

2 Kumar-Sinha C, Tomlins SA, Chinnaiyan AM. Recurrent gene fusions in prostate cancer. Nat Rev Cancer 2008;8:497-511.

3 Hermans KG, van der Korput HA, van Marion R, et al. Truncated ETV1, fused to novel tissue-specific genes, and full-length ETV1 in prostate cancer. Cancer Res 2008;68:7541-7549.

4 Attard G, Clark J, Ambroisine L, et al. Transatlantic Prostate Group. Heterogeneity and clinical significance of ETV1 translocations in human prostate cancer. Br J Cancer 2008;99:314-320.

5 Pflueger D, Rickman DS, Sboner A, et al. N-myc downstream regulated gene 1 (NDRG1) is fused to ERG in prostate cancer. Neoplasia 2009;11:804-811.

6 Laxman B, Morris DS, Yu J, et al. A first-generation multiplex biomarker analysis of urine for the early detection of prostate cancer. Cancer Res 2008;68: 645-649.

7 Sun S, Schiller JH, Gazdar AF. Lung cancer in never smokers-a different disease. Nat Rev Cancer 2007;7:778-790.

8 Mosquera JM, Mehra R, Regan MM, et al. Prevalence of TMPRSS2-ERG fusion prostate cancer among men undergoing prostate biopsy in the United States. Clin Cancer Res 2009;15:4706-4711.

9 Demichelis F, Fall K, Perner S, et al. TMPRSS2:ERG gene fusion associated with lethal prostate cancer in a watchful waiting cohort. Oncogene 2007;26:4596-4599.

10 Setlur SR, Mertz KD, Hoshida Y, et al. Estrogendependent signaling in a molecularly distinct subclass of aggressive prostate cancer. J Natl Cancer Inst 2008; 100:815-825.

11 Clark J, Merson S, Jhavar S, et al. Diversity of TMPRSS2-ERG fusion transcripts in the human prostate. Oncogene 2007;26:2667-2673. 
12 Wang J, Cai Y, Ren C, et al. Expression of variant TMPRSS2/ERG fusion messenger RNAs is associated with aggressive prostate cancer. Cancer Res 2006;66: 8347-8351.

13 Jhavar S, Reid A, Clark J, et al. Detection of TMPRSS2ERG translocations in human prostate cancer by expression profiling using GeneChip Human Exon 1.0 ST arrays. J Mol Diagn 2008;10:50-57.

14 Rickman DS, Pflueger D, Moss B, et al. SLC45A3-ELK4 is a novel and frequent erythroblast transformationspecific fusion transcript in prostate cancer. Cancer Res 2009;69:2734-2738.

15 Maher CA, Kumar-Sinha C, Cao X, et al. Transcriptome sequencing to detect gene fusions in cancer. Nature 2009;458:97-101.

16 Arora R, Koch MO, Eble JN, et al. Heterogeneity of Gleason grade in multifocal adenocarcinoma of the prostate. Cancer 2004;100:2362-2366.

17 Kobayashi M, Ishida $\mathrm{H}$, Shindo $\mathrm{T}$, et al. Molecular analysis of multifocal prostate cancer by comparative genomic hybridization. Prostate 2008;68:1715-1724.

18 Mehra R, Han B, Tomlins SA, et al. Heterogeneity of TMPRSS2 gene rearrangements in multifocal prostate adenocarcinoma: molecular evidence for an independent group of diseases. Cancer Res 2007;67:7991-7995.

19 Perner S, Demichelis F, Beroukhim R, et al. TMPRSS2:ERG fusion-associated deletions provide insight into the heterogeneity of prostate cancer. Cancer Res 2006;66:8337-8341.

20 Rajput AB, Miller MA, De Luca A, et al. Frequency of the TMPRSS2:ERG gene fusion is increased in moderate to poorly differentiated prostate cancers. J Clin Pathol 2007;60:1238-1243.

21 Mosquera JM, Perner S, Demichelis F, et al. Morphological features of TMPRSS2-ERG gene fusion prostate cancer. J Pathol 2007;212:91-101.
22 Lapointe J, Kim YH, Miller MA, et al. A variant TMPRSS2 isoform and ERG fusion product in prostate cancer with implications for molecular diagnosis. Mod Pathol 2007;20:467-473.

23 Mehra R, Tomlins SA, Shen R, et al. Comprehensive assessment of TMPRSS2 and ETS family gene aberrations in clinically localized prostate cancer. Mod Pathol 2007;20:538-544.

24 Perner S, Mosquera JM, Demichelis F, et al. TMPRSS2ERG fusion prostate cancer: an early molecular event associated with invasion. Am J Surg Pathol 2007;31:882-888.

$25 \mathrm{Tu}$ JJ, Rohan S, Kao J, et al. Gene fusions between TMPRSS2 and ETS family genes in prostate cancer: frequency and transcript variant analysis by RT-PCR and FISH on paraffin-embedded tissues. Mod Pathol 2007;20:921-928.

26 Nam RK, Sugar L, Yang W, et al. Expression of the TMPRSS2:ERG fusion gene predicts cancer recurrence after surgery for localised prostate cancer. Br J Cancer 2007;97:1690-1695.

27 Attard G, Clark J, Ambroisine L, et al. Duplication of the fusion of TMPRSS2 to ERG sequences identifies fatal human prostate cancer. Oncogene 2008;27: 253-263.

28 Yoshimoto M, Joshua AM, Cunha IW, et al. Absence of TMPRSS2:ERG fusions and PTEN losses in prostate cancer is associated with a favorable outcome. Mod Pathol 2008;21:1451-1460.

29 Gopalan A, Leversha MA, Satagopan JM, et al. TMPRSS2-ERG gene fusion is not associated with outcome in patients treated by prostatectomy. Cancer Res 2009;69:1400-1406.

30 Hofer MD, Kuefer R, Maier C, et al. Genome-wide linkage analysis of TMPRSS2-ERG fusion in familial prostate cancer. Cancer Res 2009;69:640-646.

Supplementary Information accompanies the paper on Modern Pathology website (http://www.nature.com/ modpathol) 